\title{
The All Sky Young Association (ASYA): a New Young Association
}

\author{
C. A. O. Torres ${ }^{1}$, G. R. Quast ${ }^{1}$, and D. Montes ${ }^{2}$, \\ ${ }^{1}$ Laboratório Nacional de Astrofísica/ MCT, Rua Estados Unidos 154, 37504-364, Itajubá, \\ MG, Brazil \\ email: beto@lna.br \\ ${ }^{2}$ Dpto. Astrofísica, Facultad de CC. Físicas, Universidad Complutense de Madrid, E-28040 \\ Madrid, Spain \\ email: dmontes@ucm.es
}

\begin{abstract}
To analyze the SACY (Search for Associations Containing Young stars) survey we developed a method to find young associations and to define their high probability members. These bona fide members enable to obtain the kinematical and the physical properties of each association in a proper way. Recently we noted a concentration in the $U V$ plane and we found a new association we are calling ASYA (All Sky Young Association) for its overall distribution in the sky with a total of 38 bonafide members and an estimated age of $110 \mathrm{Myr}$, the oldest young association found in the SACY survey. We present here its kinematical, space and Li distributions and its HR diagram.
\end{abstract}

Keywords. Galaxy: open clusters and associations, Stars: kinematics and dynamics, Stars: late-type

\section{Introduction}

The SACY (Search for Associations Containing Young Stars) survey was a spectroscopic effort to find southern nearby associations using as targets the possible Tycho2/HipPARCOs stars counterparts of the ROSAT All-Sky Bright Sources Catalogue (Torres et al. 2006; 2008). To analyze the SACY survey we developed a method to find young associations and to define their high probability members. These bonafide members enable to obtain the kinematical and the physical properties of each association in a proper way.

\section{ASYA (All Sky Young Association)}

Recently we noted a concentration in the $U V$ plane and we found a new association we are calling ASYA (All Sky Young Association) for its overall distribution in the sky. We also search HipPARCos catalogue for other possible members and we found a total of 38 bonafide members (including those from HipparCos and SACY). Although kinematically $(U=-15.2, V=-26.9, W=-2.8 \mathrm{~km} / \mathrm{s})$ near the Her-Lyr moving group, ASYA is definitively distinct from it and younger - we estimated an age of $110 \mathrm{Myr}$, the oldest of the young associations found in the SACY survey. A weak expansion in the X direction is present $U=<U>+k(X-<X>)$ and the solution was obtained with a $k=0.02$. We present its kinematical $(U V W)$ and space $(X Y Z)$ distributions in Fig. 1 and its Li distribution $(E W(\mathrm{Li})$ vs $V-I)$ and its $\mathrm{HR}$ diagram $\left(M_{V}\right.$ vs. $\left.V-I\right)$ in Fig. 2.

\section{Acknowledgements}

This work was supported by the Univ. Complutense de Madrid (UCM) and the Ministry of Economy and Competitiveness (MINECO) under grant AYA2011-30147-C03-02. 

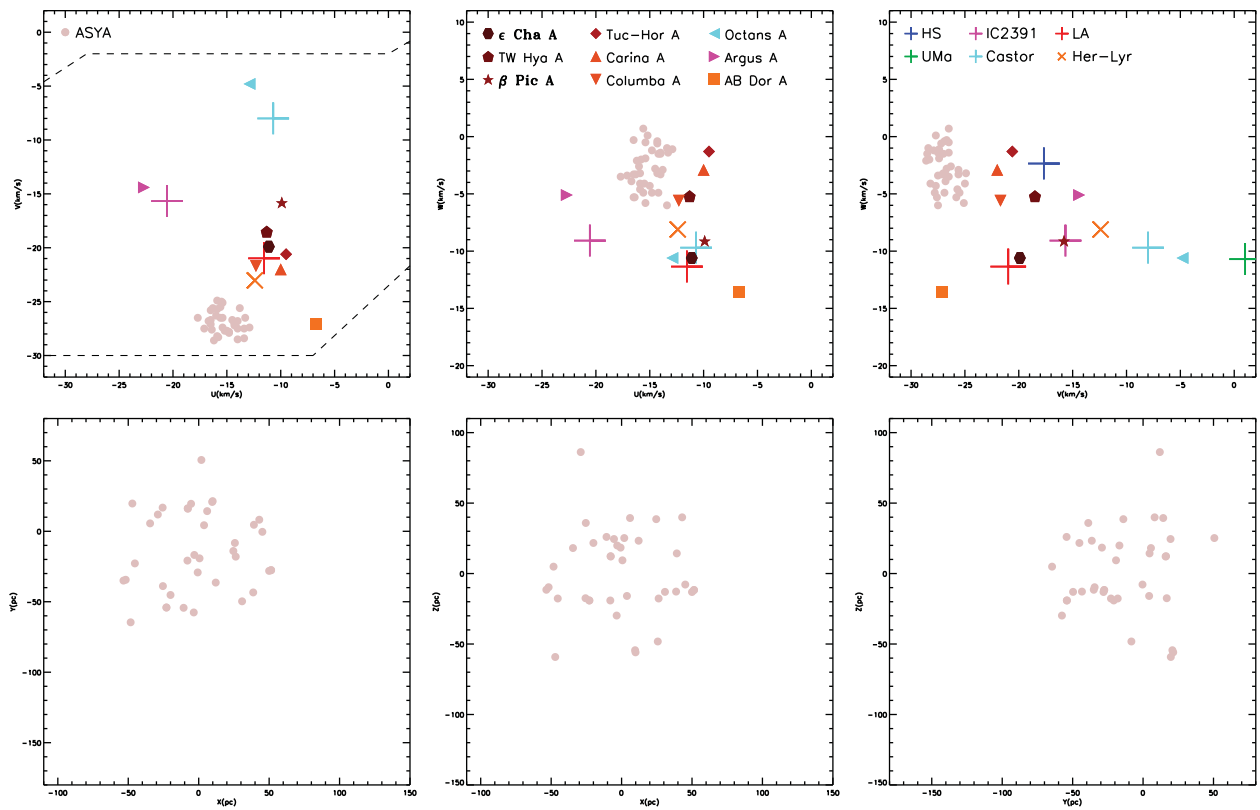

Figure 1. Upper panel: $U V W$ space for ASYA showing the well defined kinematical clustering. The position of the classical moving groups (Hyades Supercluster (HS), Ursa Major (UMa), IC2391, Castor, Local Association (LA) and Hercules-Lyra, Montes et al. 2001 and Montes 2010) and the young associations of SACY ( $\epsilon$ Cha, TW Hya, $\beta$ Pic, Tuc-Hor, Carina, Columba, Octans, Argus and AB Dor) are also presented for comparison. Note that ASYA is clearly separated from Her-Lyr and the other younger associations. Lower panel: $X Y Z$ space for ASYA.
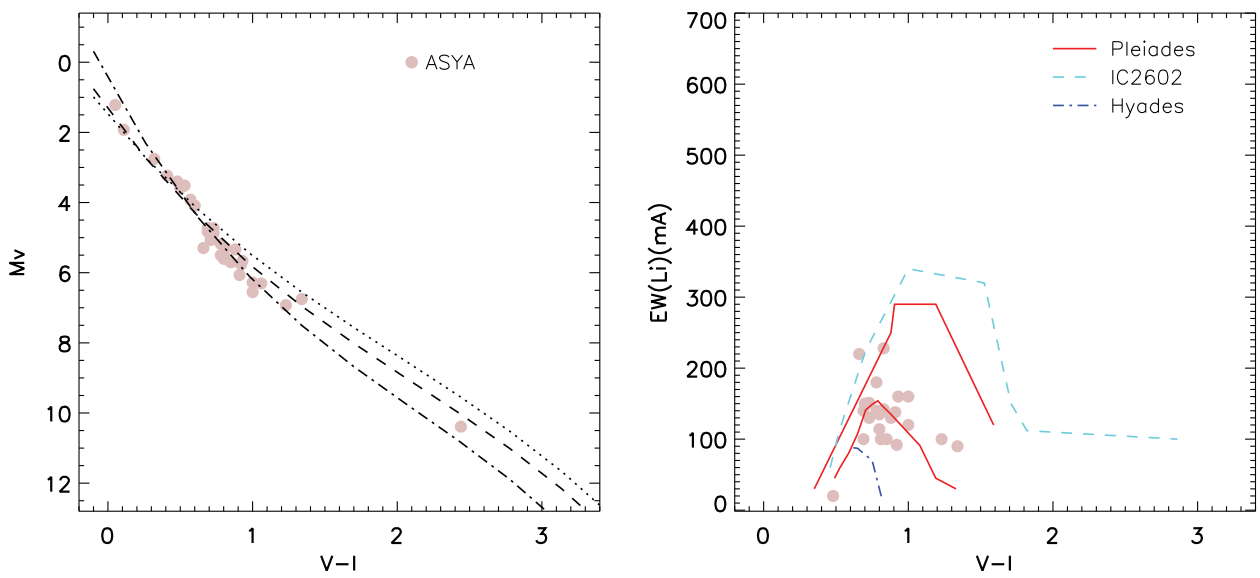

Figure 2. Left panel: The HR diagram for the proposed members of ASYA. The over-plotted curves are our ad-hoc isochrones. The upper curve is estimated as about $20 \mathrm{Myr}$ and the lower curve 110 Myr. Right panel: Lithium distribution $(E W(\mathrm{Li})$ vs $V-I)$ of ASYA compared with the Li distribution of IC 2602, Pleiades and Hyades open clusters.

\section{References}

Montes, D., López-Santiago, J., Gálvez, M. C., et al. 2001, MNRAS, 328, 45

Montes, D. 2010, IAU Special Session SpS7, Highlights of Astronomy, 15, E1

Torres, C. A. O., Quast, G. R., da Silva, L., et al. 2006, A\&BA, 460, 695

Torres, C. A. O., Quast, G. R., Melo, C. H. F., \& Sterzik, M. F. 2008, Handbook of Star Forming Regions, Volume II, 757 